\title{
Finite-rank approximations of spectral zeta residues
}

\author{
Abel B. Stern ${ }^{1}$ \\ Received: 13 March 2018 / Revised: 13 June 2018 / Accepted: 16 July 2018 / Published online: 27 July 2018 \\ (c) The Author(s) 2018
}

\begin{abstract}
We use the asymptotic expansion of the heat trace to express all residues of spectral zeta functions as regularized sums over the spectrum. The method extends to those spectral zeta functions that are localized by a bounded operator.
\end{abstract}

Keywords Zeta residues $\cdot$ Wodzicki residue $\cdot$ Dixmier trace $\cdot$ Heat kernel $\cdot$ Partial spectrum $\cdot$ Numerical asymptotics

Mathematics Subject Classification 58J42 · 58B34 · 35P20

\section{Introduction}

The spectral theory of elliptic operators presents a major connection between functional analysis and differential geometry. It provides a number of interesting relations between the spectrum with multiplicities (which is the complete unitary invariant of self-adjoint operators) and the symbol (which is directly tied to the local expression of pseudodifferential operators). Thereby, it shows how the local structure of such an operator influences its global properties, and vice versa. Of particular interest is the relation between the symbol of an elliptic operator and its spectral asymptotics that is conveyed by the spectral zeta residues.

This note is concerned with expressing the spectral zeta residues as a limit of partial sums of particular functions over the spectrum. Together with the well-known relations between the zeta residues, the symbol, and the heat expansion, this bridges a gap between the continuum set-up of spectral geometry and the finite objects in combinatorial geometry and computer science. For instance, this method allows one

The author was supported by FOM Vrij Programma No. 150.

\footnotetext{
$凶 \quad$ Abel B. Stern

a.stern@math.ru.nl; public@abelstern.nl

1 Institute for Mathematics, Astrophysics and Particle Physics, Radboud University, 6500GL Nijmegen, The Netherlands
} 
to approximate the scalar curvature on a compact Riemannian manifold using only a finite part of its Laplacian spectrum.

We will first provide some background material in Sect. 1.1 and then introduce the main topic and results of this note in Sect. 1.2.

\subsection{Background: spectral zeta residues and Weyl's law}

In 1949, Minakshisundaram and Pleijel showed [10] that the zeta function

$$
\zeta(\Delta, s) \stackrel{\text { def }}{=} \operatorname{tr} \Delta^{-s}
$$

of the Laplace operator on a compact Riemannian $d$-dimensional manifold can be meromorphically extended to the complex plane, with simple poles occurring in the points $d / 2-\mathbb{Z}_{\geq 0} \subset \mathbb{R}$. The residues at these poles (which are proportional to the so-called heat kernel coefficients) relate the spectrum of the Laplace operator, itself an isometry invariant, to other known isometry invariants of the manifold, such as its volume and scalar curvature.

More generally, an elliptic pseudodifferential operator defines a spectral zeta function, by the work of Seeley [11], and its residues relate geometric information to the operator spectrum in the following way. If $\Delta$ is a positive elliptic classical pseudodifferential operator of order $m \in \mathbb{R}_{\geq 0}$ and $k$ is any nonnegative integer, the residue at $s=(d-k) / m$ of $\zeta(\Delta, s)$ equals the Wodzicki residue

$$
\frac{1}{m} \int_{S^{*} M} \operatorname{tr} a_{-n}^{(k-d) / m}(x, \xi) \mathrm{d}^{n-1} \xi \mathrm{d} x,
$$

where $a_{-n}(x, \xi)$ is the homogenous term of order $-n$ in the decomposition of the symbol of $\Delta$ and $S^{*} M \subset T^{*} M$ is the cosphere bundle, cf. [4,8,13]. In the inversely Mellin transformed picture, the residues correspond to integrals of terms in the asymptotic expansion of the integral kernel of $\mathrm{e}^{-t \Delta}$ along the diagonal, as $t \rightarrow 0^{+}$.

One wonders which conclusions about the operator spectrum can be drawn from the zeta residues, apart from the rather opaque one provided by their definition. The most well-known result in this direction is provided by the Wiener-Ikehara theorem, which relates the first residue of the zeta function to the asymptotics of the number $N(\Lambda)$ of eigenvalues smaller than $\Lambda$. If $\alpha$ is a monotone increasing function and the zeta function

$$
\zeta(z)=\int_{1}^{\infty} \lambda^{-z} \mathrm{~d} \alpha(\lambda)
$$

converges for $\Re z>1$ and can be meromorphically extended to $\Re z \geq 1$ with a simple pole at $z=1$, then the Wiener-Ikehara theorem states that $\alpha(\Lambda) \sim \Lambda \operatorname{res}_{z=1} \zeta(z)$ as $\Lambda \rightarrow \infty$. By Seeley's results on complex powers of elliptic operators, we may apply this theory to the spectral zeta function of a degree $m$ elliptic pseudodifferential operator on a $d$-dimensional manifold. As in [10], this yields Weyl's famous law 


$$
N(\Lambda)=\operatorname{res}_{s=d / m} \zeta(\Delta, s) \Lambda^{d / m}+o\left(\Lambda^{d / m}\right)
$$

Note that this restriction only uses the first residue of the zeta function: the zeta series $\operatorname{tr} \Delta^{-s}$ is absolutely convergent for $\Re s>d / m$.

The problem of improving on the accuracy in Weyl's law has attracted much attention over the last century. That is, one wonders whether we can obtain an asymptotic expansion of $N(\Lambda)-\operatorname{res}_{s=d / m} \zeta(\Delta, s) \Lambda^{d / m}$ for specific classes of operators. However, sharp bounds that depend only on the spectral zeta function of $\Delta$ have not yet been produced, even in the well-studied case of the Laplacian on flat tori. It would seem natural that the Wiener-Ikehara result could be extended to relate the asymptotic expansion of $N(\Lambda)$ to the location of the poles of the zeta function. However, this approach is limited by the difficulties of inverse Mellin and Laplace transforms (see e.g. [1]), [2, 9.7.2]. The lower poles of the zeta function can therefore not yet be related to the asymptotics of $N(\Lambda)$. However, we will explain in the present paper how to relate their residues to the asymptotics of other functionals of the operator spectrum.

\subsection{Zeta residues as a resummation of the spectrum}

In the converse direction to Weyl's law, we ask which conclusions one can draw about the residues, given access to increasingly large finite subsets of the operator spectrum. For the first residue, Weyl's law gives rise to the Dixmier trace ${ }^{1}$ formula: if $\lambda_{0} \leq \lambda_{1} \leq \cdots$ are the eigenvalues of $\Delta$, then

$$
\operatorname{res}_{s=d / m} \zeta(\Delta, s)=\lim _{N \rightarrow \infty} \frac{\sum_{i=1}^{N} \lambda_{i}^{-d / m}}{\log N}=\lim _{\Lambda \rightarrow \infty} \frac{\sum_{\lambda<\Lambda} \lambda^{-d / m}}{\frac{d}{m} \log \Lambda}
$$

The Dixmier trace is singular: it vanishes if $\operatorname{tr} \Delta<\infty$. However, if $\Delta_{j}$ is a sequence of finite-rank operators of operator norm $\left\|\Delta_{j}\right\| \stackrel{\text { def }}{=} \Lambda_{j} \rightarrow \infty$ such that

$$
\operatorname{tr} \Delta_{j}^{-s_{0}}-\sum_{0<\lambda<\Lambda_{j}} \lambda^{-s_{0}}=o\left(\log \Lambda_{j}\right)
$$

the Dixmier trace of $\Delta^{-s_{0}}$ clearly equals $\lim _{j \rightarrow \infty} \operatorname{tr} \Delta_{j}^{-s_{0}} / \log \Lambda_{j}$. It would seem plausible that this result can be extended to further poles, i.e. that there are also functionals $c_{k}$ on the finite-rank operators such that

$$
\lim _{j \rightarrow \infty} c_{k}\left(\Delta_{j}\right)=\operatorname{res}_{s=s_{k}} \zeta(\Delta, s)
$$

if $\zeta(\Delta, s)$ has poles at $s_{0}>\cdots>s_{k}$ and the finite-rank $\Delta_{j}$ converge to $\Delta$ in some appropriate sense. The main question, then, is whether we can write these functionals down explicitly.

\footnotetext{
1 As restricted to the ideal on which this formula converges. For a treatment of the Dixmier trace on the larger ideal $L^{(1, \infty)}$, see [3].
} 
The existence of such asymptotic residue functionals is shown by our Theorem 1, and explicit expressions follow from the conditions of Propositions 1 and 2. Corollary 2 uses the theorem to improve on the convergence of the Dixmier trace formula, and Corollary 3 exhibits the resulting expression for the second pole. Finally, Theorem 2 is a simple extension of our treatment to the localized residue traces res $\operatorname{ros}_{s=s_{k}} \operatorname{tr} h \Delta^{-s}$, for any bounded operator $h$.

\section{Zeta residues as normal functionals}

We can ask how spectral zeta residues relate to finite subsets of the operator spectrum without referring to the original setting of differential geometry. We will henceforth consider the question in such generality, but we will need to restrict ourselves to operators whose zeta functions share an essential property with the Minakshisundaram-Pleijel zeta function.

Definition 1 A positive, invertible, ${ }^{2}$ self-adjoint, unbounded operator $\Delta$ with compact resolvent is said to be spectrally elliptic if the 'heat trace' $\operatorname{tr} \mathrm{e}^{-t \Delta}$ admits an asymptotic expansion $\sum_{i=0}^{\infty} c_{i} t^{-s_{i}}$ as $t \rightarrow 0^{+}$, where the $s_{i}$ are decreasingly ordered reals. We say that $\Pi=\left\{s_{i}\right\}$ is the heat spectrum of $\Delta$.

The definition implies that there is some $s_{0} \in \mathbb{R}$ such that $\Gamma(s) \operatorname{tr} \Delta^{-s}$ converges for $\Re s>s_{0}$ and can be analytically continued to a meromorphic function $\Gamma(s) \zeta(\Delta, s)$ whose poles are all simple and located in $\Pi \subset\left(-\infty, s_{0}\right]$.

This particular terminology was chosen because the motivating example of such operators are the positive elliptic differential ${ }^{3}$ operators, cf. [7,11]. Indeed, for a positive elliptic differential operator of order $m$ on a $n$-dimensional manifold, the heat spectrum is contained in $\frac{n}{m}, \frac{n-1}{m}, \ldots, \frac{1}{m}$.

Definition 1 suggests that we will in fact be technically concerned with the asymptotic heat trace coefficients $c_{i}$, and the reader who is more familiar with that terminology may rest assured that they are indeed what we are talking about. However, because zeta residues are mathematically the more general notion, the text will mainly refer to the coefficients by that name.

Our main theorem shows how the zeta residues of a spectrally elliptic operator are related to restrictions of its spectrum. We will provide the proof in Sect. 2.1.

The precise asymptotic formula for the residue $\operatorname{res}_{s=s_{k}} \zeta(\Delta, s)$ at some pole $s_{k} \in \Pi$ depends only on the location of the 'previous' poles, that is, on the set $\Pi \cap\left[s_{k}, \infty\right)$. The Dixmier trace, for instance, requires knowledge of $s_{0}$ and of the fact that no poles $s_{-1}>s_{0}$ exist. For a given finite set $\left\{s_{i}\right\}$ of such poles, all operators with zeta residues contained in this set can be considered simultaneously, hence the following definition.

Definition 2 For any finite set $\left\{s_{i}\right\}_{i=0}^{k}$ of decreasingly ordered reals, let $\mathscr{D}\left(\left\{s_{i}\right\}_{i=0}^{k}\right)$ be the set of all spectrally elliptic operators $\Delta$ whose heat spectrum $\Pi$ satisfies $\Pi \cap$

\footnotetext{
2 If not, just restrict to the complement of the kernel.

3 The classical elliptic pseudodifferential operators may have logarithmic terms in the heat expansion, leading to double poles of $\Gamma(z) \zeta(z)$ (e.g. at negative integers), and are thus excluded in general. However, see Remark 2.
} 
$\left[s_{k}, \infty\right) \subset\left\{s_{i}\right\}$, i.e. whose heat trace admits an asymptotic expansion $\sum_{i=0}^{k} c_{i} t^{-s_{i}}+$ $O\left(t^{-s_{k+1}}\right)$ as $t \rightarrow 0^{+}$, for some $s_{k+1}<s_{k}$.

If $\sigma(\Delta)$ is the (necessarily point) spectrum of some spectrally elliptic operator $\Delta$ and $F$ is any Borel measurable function, we will denote the summation of $F$ over the strictly positive eigenvalues smaller than $\Lambda$ by

$$
\operatorname{tr}_{\Lambda} F(\Delta) \stackrel{\text { def }}{=} \sum_{\Lambda>\lambda \in \sigma(\Delta)} F(\lambda) .
$$

Theorem 1 For any finite set $\left\{s_{i}\right\}_{i=0}^{k}$ of decreasingly ordered reals, there exists a function $F$ such that, for all $\Delta \in \mathscr{D}\left(\left\{s_{i}\right\}_{i=0}^{k}\right)$,

$$
\operatorname{res}_{s=s_{k}} \zeta(\Delta, s) \Gamma(s)=\lim _{\Lambda \rightarrow \infty} \varepsilon(\Lambda)^{s_{k}} \operatorname{tr}_{\Lambda} F(\Delta \varepsilon(\Lambda)),
$$

where $\varepsilon(\Lambda) \stackrel{\text { def }}{=} m \log \Lambda / \Lambda$ for any $m>s_{0}-s_{k}$.

The problem posed in Introduction is then trivially resolved by the following corollary, together with an explicit choice of $F$.

Corollary 1 Let $\left\{\Delta_{j}\right\}$ be any sequence offinite-rank positive operators of norm $\left\|\Delta_{j}\right\| \stackrel{\text { def }}{=}$ $\Lambda_{j}$ such that, as $j \rightarrow \infty, \Lambda_{j} \rightarrow \infty$ and

$$
\operatorname{tr}_{\Lambda_{j}} F\left(\Delta \varepsilon\left(\Lambda_{j}\right)\right)-\operatorname{tr} F\left(\Delta_{j} \varepsilon\left(\Lambda_{j}\right)\right)=o\left(\left(\varepsilon\left(\Lambda_{j}\right)^{-s_{k}}\right)\right.
$$

Then,

$$
\operatorname{res}_{s=s_{k}} \zeta(\Delta, s) \Gamma(s)=\lim _{j \rightarrow \infty} \varepsilon\left(\Lambda_{j}\right)^{s_{k}} \operatorname{tr} F\left(\Delta_{j} \varepsilon\left(\Lambda_{j}\right)\right)
$$

Remark 1 A uniform bound over $\mathscr{D}\left(\left\{s_{i}\right\}_{i=0}^{k}\right)$ on the rate of convergence is too much to ask: it depends on the complete spectrum of $\Delta$. However, the convergence rate will be shown to always be $O\left(\Lambda^{-1}\right)$ if the next pole is at $s_{k}-1$. For examples of arbitrary error for given $\Lambda$, see, for instance, [9,12]. For comparison, see Remark 4 on page 10 .

Remark 2 The exclusion of logarithmic terms in the heat expansion from Definition 1 corresponds to an exclusion of higher-order poles of $\Gamma(s) \zeta(\Delta, s)$. However, Theorem 1 is unchanged if higher-order poles are allowed, as long as they lie below $s_{k}$. Moreover, the approach can probably be modified to accommodate such logarithmic terms, at the expense of brevity and asymptotic rate of convergence. 


\subsection{Asymptotic series for zeta function residues}

For any spectrally elliptic operator $\Delta$, the function $\zeta(\Delta, s) \Gamma(s)$ is the Mellin transform of $\mathrm{e}^{-t \Delta}$. Therefore, the asymptotic expansion of $\mathrm{e}^{-t \Delta}$

$$
\operatorname{tr} \mathrm{e}^{-t \Delta} \sim \sum_{i=0}^{k} c_{i} t^{-s_{i}}+o\left(t^{-s_{k}}\right)
$$

has coefficients

$$
c_{i}=\operatorname{res}_{s=s_{i}} \zeta(\Delta, s) \Gamma(s)
$$

The proof of Theorem 1 relies on the asymptotic expansion of a Mellin convolution integral with $\operatorname{tr} \mathrm{e}^{-t \Delta}$. This strategy was inspired by the spectral action principle of [5, Thm 1.145] and the approach of [1]. However, here we have specifically constructed the function we convolve with such that its first moments vanish, which allows us to recover the zeta residue in the leading term.

Proof of Theorem 1 The proof proceeds in two elementary steps. First, we show (Proposition 1) that for suitable functions $f$ one has

$$
\int_{0}^{\infty} \operatorname{tr} \mathrm{e}^{-\varepsilon t \Delta} f(t)=c_{k} \varepsilon^{-s_{k}}+O\left(\varepsilon^{-s_{k+1}}\right)
$$

as $\varepsilon \rightarrow 0$. This part requires the asymptotic expansion of tr $\mathrm{e}^{-t \Delta}$, implying in particular that the poles of $\Gamma(s) \zeta(\Delta, s)$ must be simple and discrete (i.e. $\Delta$ has simple dimension spectrum disjoint with the negative integers) and lie on the real line.

Then, we use the Laplace transform $F$ to rewrite the integral as a trace

$$
\int_{0}^{\infty} \operatorname{tr} \mathrm{e}^{-\varepsilon t \Delta} f(t) \mathrm{d} t=\operatorname{tr} F(\varepsilon \Delta),
$$

and we prove in Proposition 2 that there is a choice $\varepsilon(\Lambda)=\Lambda^{-1} \log \Lambda^{m}$ such that $F$ decays as

$$
\left(\operatorname{tr}-\operatorname{tr}_{\Lambda}\right) F(\Delta \varepsilon(\Lambda))=O\left(\varepsilon^{-s_{k+1}}\right)
$$

so that we obtain the useful asymptotic behaviour

$$
\operatorname{tr}_{\Lambda} F(\Delta \varepsilon(\Lambda))=c_{k} \varepsilon(\Lambda)^{-s_{k}}+O\left(\varepsilon^{-s_{k+1}}\right)
$$

This second part relies on the finite summability of $\Delta$, that is, on the fact that $\operatorname{tr} \mathrm{e}^{-t \Delta}=$ $O\left(t^{-s_{0}}\right)$ as $t \rightarrow 0^{+}$.

The following asymptotic estimate is completely straightforward. 
Proposition 1 Let $f$ be a piecewise continuous function supported in $(1, \infty)$ that is $O\left(t^{-m}\right)$ for all $m \in \mathbb{R}$ towards $\infty$. Let $\Delta \in \mathscr{D}\left(\left\{s_{i}\right\}_{i=0}^{k}\right)$. Then, the Mellin convolution integral of $f$ with $\operatorname{tr} e^{-t \Delta}$ has an asymptotic expansion

$$
\int_{0}^{\infty} \operatorname{tr} e^{-\varepsilon t \Delta} f(t) d t=\sum_{i=0}^{k} c_{i} \varepsilon^{-s_{i}} \int_{0}^{\infty} t^{-s_{i}} f(t) d t+o\left(t^{-s_{k}}\right)
$$

Thus, if we simply choose $f$ so that its moments $\int_{0}^{\infty} t^{-s_{i}} f(t) \mathrm{d} t$ vanish for $i \neq k$ and normalize it so that $\int_{0}^{\infty} t^{-s_{k}} f(t) \mathrm{d} t=1$, we gain access to the coefficient $c_{k}$.

By asymptotic decay of such a function $f$ towards $\infty$, it has absolutely convergent Laplace transform $F$. As $\left|f(t) \operatorname{tr} \mathrm{e}^{-\varepsilon t \Delta}\right|$ is absolutely integrable as well, we can apply the Fubini-Tonelli theorem to obtain

$$
\begin{aligned}
\int_{0}^{\infty} \operatorname{tr} \mathrm{e}^{-\varepsilon t \Delta} f(t) \mathrm{d} t & =\sum_{\lambda \in \sigma(\Delta)} \int_{0}^{\infty} \mathrm{e}^{-\lambda \varepsilon t} f(t) \mathrm{d} t \\
& =\operatorname{tr} F(\Delta \varepsilon),
\end{aligned}
$$

where $\sigma(\Delta)$ is the spectrum (with multiplicities) of $\Delta$. Therefore, Proposition 1 leads us to the conclusion that

$$
\operatorname{tr} F(\varepsilon \Delta)=c_{k} \varepsilon^{-s_{k}}+O\left(\varepsilon^{-s_{k+1}}\right)
$$

The following proposition shows that we retain the same asymptotics if we replace the trace of $F(\varepsilon \Delta)$ by a sum over a finite part of the spectrum, provided we scale $\varepsilon$ accordingly.

Proposition 2 If $f$ and $\Delta$ are as in Proposition 1 and additionally

$$
\int_{0}^{\infty} t^{-s_{i}} f(t) d t=0 \quad(\text { for all } i<k)
$$

then the Laplace transform F satisfies

$$
\sum_{\Lambda<\lambda \in \sigma(\Delta)} F\left(\lambda \log \Lambda^{m} / \Lambda\right)=o\left(\left(\log \Lambda^{m} / \Lambda\right)^{-s_{k}}\right)
$$

for all $m>s_{0}-s_{k}$.

Proof By the fact that supp $f \subset[1, \infty)$, its Laplace transform decays as $F(s)=$ $O\left(s^{-1} \mathrm{e}^{-s}\right)$ towards $\infty$. As $\sum_{\lambda>\Lambda} \mathrm{e}^{-\varepsilon(\lambda-\Lambda) t}$ is monotone decreasing in $t$, we see that

$$
\sum_{\lambda>\Lambda} F(\varepsilon \lambda)=O\left(\sum_{\lambda>\Lambda}(\varepsilon \Lambda)^{-1} \mathrm{e}^{-\varepsilon \lambda}\right)=O\left((\varepsilon \Lambda)^{-1} \mathrm{e}^{-\varepsilon \Lambda} \sum_{\lambda>\Lambda} \mathrm{e}^{-\varepsilon(\lambda-\Lambda) t}\right)
$$


for any $t \leq 1$, as $\Lambda \varepsilon \rightarrow \infty$. This, in turn, is $O\left((\varepsilon \Lambda)^{-1} \mathrm{e}^{-\varepsilon \Lambda(1-t)} \varepsilon^{-s_{0}}\right)$. If, then, $\varepsilon(\Lambda)=m \Lambda^{-1} \log \Lambda$ for any $m>\left(s_{0}-s_{k}\right)$, the remainder $\sum_{\lambda>\Lambda} F(\varepsilon \lambda)$ will be $o\left(\varepsilon^{-s_{k}}\right)$.

This completes the proof of Theorem 1 that started on page 5 .

\subsection{Explicit formulas for the first two poles}

One reason to look for series that converge to zeta residues is to obtain geometric information from finite-dimensional approximations of the Laplacian on a Riemannian manifold. For instance, if $\Delta$ is the Laplacian, the first two residues are proportional to the volume and the total scalar curvature, respectively.

The first pole is a classical object of study. Its residue is expressed by Dixmier's singular trace and is used, for instance, for the zeta regularization of divergent series. It is connected to counting asymptotics by the Wiener-Ikehara theorem, and for a Laplace operator on a compact Riemannian manifold, it is proportional to the volume.

Our Theorem 1 provides the following formula for the first residue.

Corollary 2 If $\Delta$ is a spectrally elliptic operator with heat spectrum bounded from above by $s_{0} \in \mathbb{R}$, then if $\varepsilon(\Lambda)=\log \Lambda / \Lambda$,

$$
\operatorname{res}_{s=s_{0}} \zeta(\Delta, s) \Gamma\left(s_{0}\right)=\lim _{\Lambda \rightarrow \infty} \frac{\varepsilon(\Lambda)^{s_{0}}}{e \Gamma\left(1-s_{0}, 1\right)} \operatorname{tr}_{\Lambda} \frac{e^{-\Delta \varepsilon(\Lambda)}}{1+\Delta \varepsilon(\Lambda)}
$$

Proof Use $f=[t \geq 1] \mathrm{e}^{-t}$, with Laplace transform $F(s)=\mathrm{e}^{-1-s} /(1+s)$, in Proposition 1 . Then, divide by $\int_{0}^{\infty} t^{-s_{0}} f(t) \mathrm{d} t$ to satisfy the conditions of Proposition 2.

Remark 3 The present series converges faster in general than the logarithmic trace suggested by Weyl's asymptotic formula; the remainder is $O\left(\varepsilon^{s_{0}-s_{1}}\right)=$ $O\left(\Lambda^{s_{1}-s_{0}}(\log \Lambda)^{s_{0}-s_{1}}\right)$, whereas for e.g. the Laplacian on the circle the logarithmic trace of $\Delta^{-1 / 2}$ convergences only as $\sum_{n=1}^{N} \frac{1}{n \log N}-1=\frac{\gamma+O\left(N^{-1}\right)}{\log N}$.

Now, the second pole is of particular interest because it is the first pole for which no asymptotic residue formula was previously known and because it provides a way to calculate the total scalar curvature of a Riemannian manifold from partial spectra of the Laplacian.

Corollary 3 If $\Delta$ is a spectrally elliptic operator with heat spectrum $\left\{s_{0}, s_{0}-1, \ldots\right\}$, then if $\varepsilon(\Lambda)=2 \log \Lambda / \Lambda$,

$$
\operatorname{res}_{s=s_{1}} \zeta(\Delta, s) \Gamma(s)=\lim _{\Lambda \rightarrow \infty} \frac{\varepsilon(\Lambda)^{s_{0}-1}}{e \Gamma\left(2-s_{0}, 1\right)} \operatorname{tr}_{\Lambda}\left(\frac{2^{s_{0}} e^{-2 \Delta \varepsilon(\Lambda)}}{1+2 \Delta \varepsilon(\Lambda)}-\frac{e^{-\Delta \varepsilon(\Lambda)}}{1+\Delta \varepsilon(\Lambda)}\right)
$$

Proof Use $f=[t \geq 1] \mathrm{e}^{-t}-2^{s_{0}-1}[t \geq 2] \mathrm{e}^{-t / 2}$, which clearly satisfies the conditions of Proposition 1 and has vanishing moment $\int_{0}^{\infty} t^{-s_{0}} f(t) \mathrm{d} t$. Therefore, if we divide 
by $\int_{0}^{\infty} t^{1-s_{0}} f(t) \mathrm{d} t$, the result will be as in Proposition 2. The Laplace transform of $f$ is $F(s)=\mathrm{e}^{-1-s} /(1+s)-2^{s_{0}} \mathrm{e}^{-1-2 s} /(1+2 s)$.

Example 1 (The total scalar curvature of the sphere $S^{2}$ ) Let $F$ be the residue functional given by

$$
F(s):=\left(\frac{\exp (-s)}{1+s}-\frac{2 \exp (-2 s)}{1+2 s}\right)
$$

let $q_{n}=n(n+1)$ be the $n$th eigenvalue of the Laplacian on the sphere and let $p_{n}=2 n+1$ be its multiplicity. As before, write $\varepsilon_{m}=2 q_{m}^{-1} \log q_{m}$. We will use the Euler-Maclaurin formula to estimate the series from Corollary 3,

$$
S(m):=\sum_{n=0}^{m} p_{n} F\left(\varepsilon_{m} q_{n}\right)
$$

Sufficient accuracy will turn out to require only the first nontrapezoidal correction term. The Euler-Maclaurin estimate reads

$$
S(m)=\int_{0}^{m} p_{n} F\left(\varepsilon_{m} q_{n}\right) \mathrm{d} n+\left.\left(\frac{1}{2}+\frac{B_{2}}{2} \frac{\partial}{\partial n}\right) p_{n} F\left(\varepsilon_{m} q_{n}\right)\right|_{0} ^{m}+R_{3}(m),
$$

where $B_{2}=\frac{1}{6}$, and we will employ the usual rough bound

$$
\left|R_{3}(m)\right| \leq \frac{\zeta(3)}{(2 \pi)^{3}} m \sup _{0 \leq n \leq m}\left|\frac{\partial^{3}\left(p_{n} F\left(\varepsilon_{m} q_{n}\right)\right)}{\partial n^{3}}\right|
$$

The integral in the first term can be taken analytically,

$\int_{0}^{m} p_{n} F\left(\varepsilon_{m} q_{n}\right) \mathrm{d} n=\int_{0}^{q_{m}} F\left(q, \varepsilon_{m}\right) \mathrm{d} q=e \varepsilon_{m}^{-1}\left(E_{1}\left(1+2 \varepsilon_{m} q_{m}\right)-E_{1}\left(1+\varepsilon_{m} q_{m}\right)\right)$,

and is $O\left(q_{m}^{-2} \log \left(1+q_{m} /\left(2 \log q_{m}\right)\right)\right.$.

Because $F(s)=O\left(s^{-1} \mathrm{e}^{-s}\right)$ as $s \rightarrow \infty$, the order zero boundary term is just $\frac{1}{2}+\frac{1}{2} p_{m} F\left(\varepsilon_{m} q_{m}\right)=\frac{1}{2}+O\left(p_{m} q_{m}^{-2} / \log q_{m}\right)$.

The first correction term to the trapezoidal approximation equals

$$
\frac{1}{12}\left(-2+2 F\left(\varepsilon_{m} q_{m}\right)+\varepsilon_{m} p_{m}^{2} F^{(1)}\left(\varepsilon_{m} q_{m}\right)\right)=-\frac{1}{6}+O\left(q_{m}^{-2} \log q_{m}\right) .
$$

Finally, the third derivative of $p_{n} F\left(\varepsilon_{m} q_{n}\right)$ with respect to $n$, whose supremum appears in the error estimate, is

$$
12 \varepsilon_{m} F^{(1)}\left(\varepsilon_{m} q_{n}\right)+12 \varepsilon_{m}^{2} p_{n}^{2} F^{(2)}\left(\varepsilon_{m} q_{n}\right)+\varepsilon_{m}^{3} p_{n}^{4} F^{(3)}\left(\varepsilon_{m} q_{n}\right) .
$$


All derivatives of $F$ are bounded on $[0, \infty)$, so these terms are all $O\left(q_{m}^{-1} \log q_{m}\right)$ uniformly in $n$. Thus, $R_{3}(m)=O\left(m^{-1} \log q_{m}\right)$.

Combining these facts with Corollary 3 , we see that the nonleading residue for the zeta function $\zeta_{S^{2}}$ is

$$
\operatorname{res}_{s=0} \zeta_{S^{2}}^{-s}=\lim _{m \rightarrow \infty}\left(\frac{1}{2}-\frac{1}{6}+O\left(m^{-1} \log q_{m}\right)\right)=\frac{1}{3}=\frac{2 \cdot 4 \pi}{6 \cdot(4 \pi)^{d / 2}}
$$

Of course, one could apply Euler-Maclaurin directly to the sum that defines the heat trace on $S^{2}$; since the corresponding limits $m \rightarrow \infty$ can termwise be identified as power series in $t$, one can reproduce the asymptotics as $t \rightarrow 0$ afterwards. In the present formulation, however, we need only take a single limit in $m$ and have expressed the residue as a single universal series in the eigenvalues, with an asymptotic bound on the error over all of $\mathscr{D}\left(s_{0}, s_{0}-1\right)$.

\section{Localization}

If $\Delta$ is spectrally elliptic, Theorem 1 allows us to calculate its zeta residues as a series in its eigenvalues. However, the classical theory of elliptic pseudodifferential operators assigns to them not just the total zeta residues, but rather a set of zeta densities. To be precise, the somewhat simpler situation for elliptic differential operators is as follows.

Let $M$ be a Riemannian manifold of dimension $n$ equipped with a smooth Hermitian vector bundle $E$, and let $\Delta$ be a positive, self-adjoint, elliptic differential operator of order $m>0$, acting inside the Hilbert space of square-integrable sections of $E$. Then, the following localized asymptotics are available.

1. The operator $\mathrm{e}^{-t \Delta}$ is given by a smooth kernel $k(x, y, t): E_{y} \rightarrow E_{x}$, and as $t \rightarrow 0^{+}$, its restriction to the diagonal admits an asymptotic expansion in smooth sections $k_{j}$ of End $E$,

$$
k(x, x, t) \sim \sum_{j=0}^{\infty} k_{j}(x) t^{(j-n) / m} .
$$

2. For any continuous section $h$ of End $E$ and any $j \geq 0$, the following residues exist and satisfy

$$
\operatorname{res}_{s=(j-n) / m} \Gamma(s) \operatorname{tr} h \Delta^{-s}=\int_{M} \operatorname{tr}\left(h(x) k_{j}(x)\right) \mathrm{d} \mathrm{vol}_{M}(x) .
$$

We say that $h$ localizes the residue trace, and we are interested in expressing the localized residues in a fashion similar to Theorem 1 . We will solve this localization problem in a slightly more general setting, along the lines of the previous treatment of the zeta function residues. 
Let $\Delta$ be a spectrally elliptic operator on a Hilbert space $\mathscr{H}$ and let $h \in \mathscr{B}(\mathscr{H})$ be bounded. Then, there exist $s_{0}>\cdots>s_{k+1}$ such that, as $t \rightarrow 0^{+}$,

$$
\operatorname{tr} h \mathrm{e}^{-t \Delta}-\sum_{i=0}^{k} t^{-s_{k}} c_{i}(h)=O\left(t^{-s_{k+1}}\right)
$$

As before, let $\left\{s_{i}\right\}_{i=1}^{k}$ be a set of decreasingly ordered reals and let $F$ be the Laplace transform of a piecewise continuous function $f$ supported in $[1, \infty)$, which is $O\left(t^{-m}\right)$ for all $m \in \mathbb{R}$ towards $\infty$, satisfies $\int_{0}^{\infty} t^{-s_{i}} f(t) \mathrm{d} t=0$ for all $i<k$, and is normalized to satisfy $\int_{0}^{\infty} t^{-s_{k}} f(t) \mathrm{d} t=1$.

Theorem 2 Let $\Delta \in \mathscr{D}\left(\left\{s_{i}\right\}_{i=1}^{k}\right)$ be a spectrally elliptic operator on a Hilbert space $\mathscr{H}$ and let $h \in \mathscr{B}(\mathscr{H})$ be bounded. Then, for any orthonormal basis $\left\{\phi_{\lambda}\right\}_{\lambda \in \sigma(\Delta)}$ of $\mathscr{H}$ diagonalizing $\Delta$, we have

$$
\operatorname{res}_{s=s_{k}} \Gamma(s) \operatorname{tr} h \Delta^{-s}=\lim _{\Lambda \rightarrow \infty} \varepsilon(\Lambda)^{s_{k}} \sum_{\Lambda>\lambda \in \sigma(\Delta)}\left\langle h \phi_{\lambda}, \phi_{\lambda}\right\rangle F(\lambda \varepsilon(\Lambda)),
$$

where $\varepsilon(\Lambda) \stackrel{\text { def }}{=} \Lambda^{-1} \log \Lambda^{m}$ for any $m>s_{k}-s_{0}$.

Proof As before, Proposition 1 holds for any such function $f$. That is,

$$
\int_{0}^{\infty} \operatorname{tr} h \mathrm{e}^{-\varepsilon t \Delta} f(t) \mathrm{d} t=c_{k}(h) \varepsilon^{-s_{k}}+\|h\|_{\mathrm{op}} O\left(\varepsilon^{-s_{k+1}}\right) .
$$

Moreover, $\sum_{\lambda \in \sigma(\Delta)}\left|f(t) \mathrm{e}^{-t \lambda}\left\langle h \phi_{\lambda}, \phi_{\lambda}\right\rangle\right| \leq|f(t)|\|h\|_{\text {op }}$ tr $\mathrm{e}^{-t \Delta}$ is in $L^{1}(0, \infty)$. Therefore, by dominated convergence,

$$
\int_{0}^{\infty} \operatorname{tr} h \mathrm{e}^{-\varepsilon t \Delta} f(t) \mathrm{d} t=\sum_{\lambda \in \sigma(\Delta)}\left\langle h \phi_{\lambda}, \phi_{\lambda}\right\rangle F(\varepsilon \lambda)
$$

and by Proposition 2, the rest term decays like

$$
\sum_{\Lambda<\lambda \in \sigma(\Delta)} F(\lambda \varepsilon(\Lambda))\left\langle h \phi_{\lambda}, \phi_{\lambda}\right\rangle=\|h\|_{\mathrm{op}} O\left((\varepsilon(\Lambda))^{-s_{k+1}}\right) .
$$

We have $\operatorname{res}_{s=s_{k}} \Gamma(s) \operatorname{tr} h \Delta^{-s}=c_{k}(h)$, and the conclusion follows.

\section{Final remarks and suggestions}

The original motivation behind this note was to confirm the point of view that finiterank cut-offs of spectral triples can carry geometric information in noncommutative geometry. The following remarks all proceed in that direction. 
Remark 4 Our Theorem 1 provides a partial counterweight to a classical result by Colin de Verdière [12]. On the one hand, he showed that a finite set of Laplace eigenvalues carries no information on the metric if no pointwise bounds on the sectional curvature are imposed. On the other hand, we now see that for each metric and each desired accuracy, there is a bound $\Lambda$ such that the set of eigenvalues smaller than $\Lambda$, with multiplicity, allow computation of all zeta residues (and hence, of the associated global invariants of the metric) up to that accuracy. The local version, using Theorem 2, of this statement is that for each metric and each $\varepsilon$ there is a finite-rank projection $P_{\Lambda}=[0, \Lambda]\left(\Delta_{g}\right)$ such that the cut-off matrix $P_{\Lambda} \Delta_{g} P_{\Lambda}$ together with the cut-off $P_{\Lambda} C(M) P_{\Lambda}$ of the function algebra yields the residues of $\operatorname{tr} a \Delta^{-s}$ up to an error of $\|a\| \varepsilon$.

Remark 5 The method of Theorem 2 may be applied to obtain a reasonable definition of scalar curvature in finite-dimensional noncommutative geometry. Let $(A, H, D)$ be a spectral triple such that $D^{2}$ is spectrally elliptic with heat spectrum $\{(j-n) / 2\}_{j \in \mathbb{Z}_{>0}}$. In [5], the scalar curvature functional of such a spectral triple was defined to be the map

$$
a \mapsto R(a) \stackrel{\text { def }}{=} \operatorname{res}_{s=n-2} \Gamma(s) \operatorname{tr} a D^{-s} .
$$

If $H$ is finite-dimensional but a dimension spectrum of $D$ is specified in advance (e.g. by modelling considerations), this residue always vanishes and Theorem 2 suggests it should perhaps be replaced by

$$
a \mapsto R_{\Lambda}(a) \stackrel{\text { def }}{=} \sum_{\lambda \in \sigma\left(D^{2}\right)}\left\langle a \phi_{\lambda}, \phi_{\lambda}\right\rangle \frac{F\left(\lambda \log \Lambda^{m} / \Lambda\right)}{\left(\log \Lambda^{m} / \Lambda\right)^{n / 2-1}},
$$

where $\Lambda \stackrel{\text { def }}{=}\left\|D^{2}\right\|^{\rho}$ for any $0<\rho<1,\left\{\phi_{\lambda} \mid D^{2} \phi_{\lambda}=\lambda \phi_{\lambda}\right\}$ is an orthonormal basis of $H$ and $F$ is as in Corollary 3. Mutatis mutandis, the same applies to the volume and other spectral invariants.

Remark 6 The calculation of the residues of localized zeta functions, Theorem 2, can be combined with the local index formula of Connes and Moscovici [6] for the Chern character of the Fredholm module associated to a spectral triple $(A, H, D)$, in order to estimate some KK-theoretic index pairings numerically. If the square of the operator $D$ is spectrally elliptic, the index pairings can be expressed as a series in the spectrum of $D^{2}$ and the coefficients $\operatorname{tr} \pi_{\lambda} a_{0}\left[D, a_{1}\right]^{\left(k_{1}\right)} \cdots\left[D, a_{n}\right]^{\left(k_{n}\right)} \pi_{\lambda}$, where $\pi_{\lambda}$ projects onto the eigenspace associated with the eigenvalue $\lambda$, and the commutators are defined recursively as $[D, a]^{(k+1)} \stackrel{\text { def }}{=}\left[D^{2},[D, a]^{(k)}\right]$ and $a_{0}, \ldots, a_{n} \in A$. A similar statement holds in the presence of a grading.

Open Access This article is distributed under the terms of the Creative Commons Attribution 4.0 International License (http://creativecommons.org/licenses/by/4.0/), which permits unrestricted use, distribution, and reproduction in any medium, provided you give appropriate credit to the original author(s) and the source, provide a link to the Creative Commons license, and indicate if changes were made. 


\section{References}

1. Aramaki, J.: An extension of the Ikehara Tauberian theorem and its application. Acta Math. Hungar. 71(4), 297-326 (1996). https://doi.org/10.1007/BF00114420

2. Berger, M.: A Panoramic View of Riemannian Geometry. Springer, Berlin (2003). https://doi.org/10. 1007/978-3-642-18245-7

3. Carey, A.L., Rennie, A., Sedaev, A., Sukochev, F.: The Dixmier trace and asymptotics of zeta functions. J. Funct. Anal. 249(2), 253-283 (2007). https://doi.org/10.1016/j.jfa.2007.04.011

4. Connes, A.: Noncommutative Geometry. Academic Press Inc, San Diego (1994)

5. Connes, A., Marcolli, M.: Noncommutative Geometry, Quantum Fields and Motives. American Mathematical Society Colloquium Publications, vol. 55. American Mathematical Society, Providence, RI; Hindustan Book Agency, New Delhi (2008)

6. Connes, A., Moscovici, H.: The local index formula in noncommutative geometry. Geom. Funct. Anal. 5(2), 174-243 (1995). https://doi-org.ru.idm.oclc.org/10.1007/BF01895667

7. Duistermaat, J.J., Guillemin, V.W.: The spectrum of positive elliptic operators and periodic bicharacteristics. Invent. Math. 29(1), 39-79 (1975). https://doi.org/10.1007/BF01405172

8. Gilkey, P.B.: Invariance Theory, the Heat Equation, and the Atiyah-Singer Index Theorem. Studies in Advanced Mathematics, 2nd edn. CRC Press, Boca Raton (1995)

9. Lohkamp, J.: Discontinuity of geometric expansions. Comment. Math. Helv. 71(2), 213-228 (1996). https://doi.org/10.1007/BF02566417

10. Minakshisundaram, S., Pleijel, A.: Some properties of the eigenfunctions of the Laplace-operator on Riemannian manifolds. Can. J. Math. 1, 242-256 (1949)

11. Seeley, R.T.: Complex powers of an elliptic operator. In: Singular Integrals (Proc. Sympos. Pure Math., Chicago, Ill., 1966), pp. 288-307. Amer. Math. Soc., Providence, R.I. (1967)

12. Colin de Verdière, Y.: Construction de laplaciens dont une partie finie du spectre est donnée. Ann. Sci. École Norm. Sup. (4) 20(4), 599-615 (1987). http://www.numdam.org/item?id=ASENS_1987_ 4_20_4_599_0

13. Wodzicki, M.: Noncommutative residue. I. Fundamentals. In: $K$-Theory, Arithmetic and Geometry (Moscow, 1984-1986), Lecture Notes in Math., vol. 1289, pp. 320-399. Springer, Berlin (1987). https:// doi.org/10.1007/BFb0078372 